Speckle Classification for Sensorless Freehand 3D Ultrasound

P. Hassenpflug, R. W. Prager,

G. M. Treece and A. H. Gee

CUED/F-INFENG/TR 513

March 2005

Cambridge University Engineering Department

Trumpington Street

Cambridge CB2 1PZ

England

Email: \{ph305,rwp,gmt11, ahg\}@eng.cam.ac.uk 



\title{
Speckle Classification for Sensorless Freehand 3D Ultrasound
}

\author{
Peter Hassenpflug, Richard Prager, Graham Treece and Andrew Gee \\ University of Cambridge Department of Engineering \\ Trumpington Street, Cambridge CB2 1PZ
}

\begin{abstract}
Despite being a valuable tool for volume measurement and the analysis of complex geometry, the need for an external position sensor is holding up the clinical exploitation of freehand three-dimensional ultrasound. Some sensorless systems have been developed, using speckle decorrelation for out-of-plane distance estimation, but their accuracy is still not as good as that of sensor-based systems. Here, we examine the widely held belief that accuracy can be improved by limiting the distance measurements to patches of ultrasound data containing fully developed speckle. Without speckle detection, we observe that scan separation is systematically underestimated by $33.1 \%$ in biological tissue. We describe a number of speckle detectors and show that they reduce the underestimate to about $25 \%$. We conclude that speckle classification can improve the quality of distance estimation, but not sufficiently to achieve accurate, metric reconstruction of the insonified volume.
\end{abstract}

\section{Speckle-based distance measurement}

Freehand three-dimensional (3D) ultrasound (US), in which B-scan slices and probe trajectory are simultaneously recorded, provides a valuable clinical tool for volume measurement and the analysis of complex geometry [8, 9, 21, 24]. However, the need for an add-on position sensor is inconvenient in a clinical environment. The goal is thus to use less intrusive techniques for probe tracking without compromising the spatial accuracy of the overall system. The development of accurate, sensorless measurement techniques is key to improving the clinical utility of freehand 3D US.

Position sensorless freehand 3D US makes use of the fact that the beam of the US scanner is several millimetres wide in the elevational ${ }^{1}$ direction, even at the focus. Each pixel in the scan image is sensitive to back-scattered echoes from a roughly ellipsoidal volume known as the resolution cell [1]. As illustrated in the left part of Fig. 1, the resolution cells in neighbouring B-scans overlap and, as a result, there is a statistical link between the corresponding echoes. To model this link, two alternative theories have been developed, one based on correlation of the echo intensities [2, 11, 18, 29], the other on linear regression [26]. For scans comprising fully developed speckle, Prager et al. [26] demonstrated the equivalence of the two theories.

Speckle-based distance measurement exploits the fact that there is a roughly Gaussian relationship between the correlation (or regression gradient) of the echo envelope intensities

\footnotetext{
${ }^{1}$ The following standard nomenclature is used to refer to the principal directions in ultrasound imaging. The axial direction is in the direction of wave propagation, from the transducer into the skin. The lateral direction is the other principal direction in the plane of the B-scan. The elevational direction is perpendicular to the plane of the B-scan.
} 

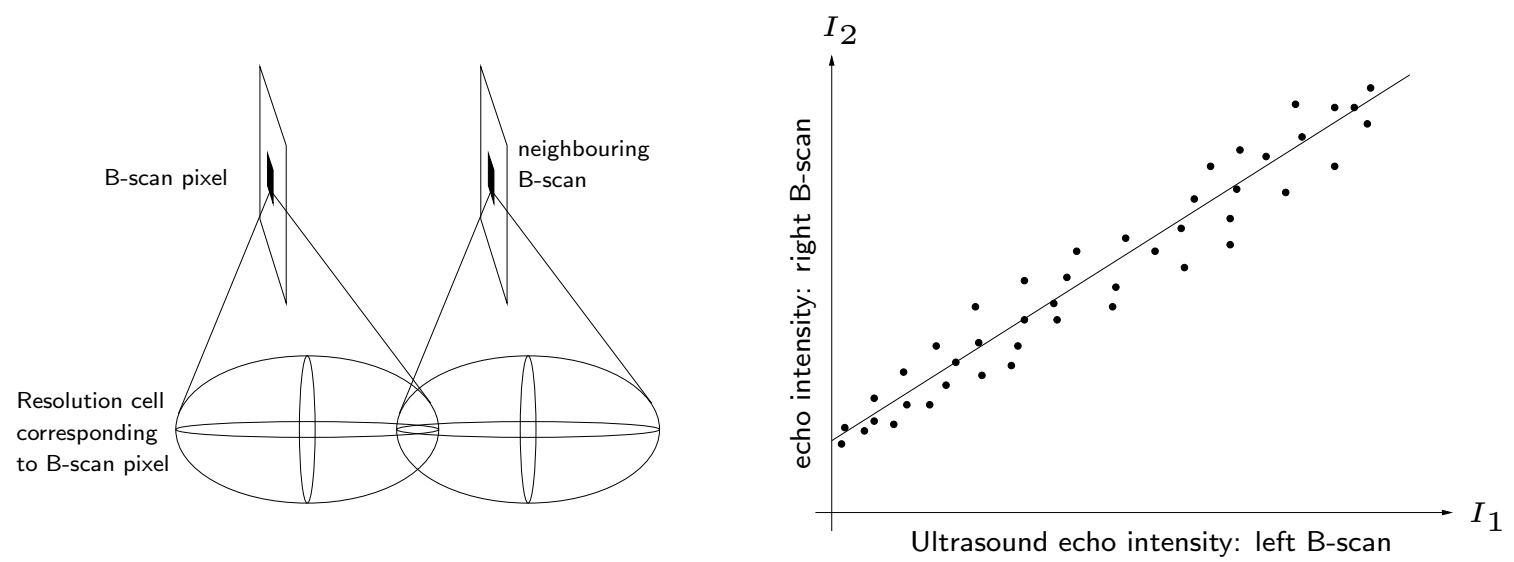

Figure 1: Left: Overlapping resolution cells from neighbouring B-scans. Right: Illustration of the relationship between the uncompressed echo envelope intensity from corresponding resolution cells in neighbouring B-scans.

of a pair of patches of speckle, and their distance apart [11]. The precise shape of this relationship depends on the beam profile, but it is usually of the form illustrated in the right part of Fig. 2. For a Gaussian-shaped resolution cell with a width characterised by the standard deviation $\sigma$, the following two steps can be performed to determine the distance between two B-scans.

1. Perform linear regression (or correlation) between the back-scattered envelope intensities in corresponding patches of the two scans.

2. Use the gradients $b$ of the best fit lines (or equivalently Pearson's correlation coefficient $\rho$ ) and the standard deviation of the US resolution cells to find the distances $d$ between the B-scans at various points using the relationship

$$
b=\exp \left(-\frac{d^{2}}{2 \sigma^{2}}\right) .
$$

Two prerequisites have to be fulfilled in order to achieve accurate distance measurements.

1. The theory for speckle-based distance measurement is only valid for intensity echoes resulting from fully developed speckle.

2. The local width of the non-stationary resolution cell must be known.

The goal of this paper is to advance the accuracy of speckle-based out-of plane distance measurement. To this end, we employ highly localised models of the shape of the resolution cell, which varies across the B-scan for reasons described in [33]. We also investigate the ability of various speckle detectors to select appropriate data for regression or correlation. We are not aware of any published papers that evaluate the short range performance of these algorithms in the way we report here.

The organisation of this paper is as follows. Section 2 presents the model for the local shape of the resolution cell and its training from parallel B-scans with known elevational 


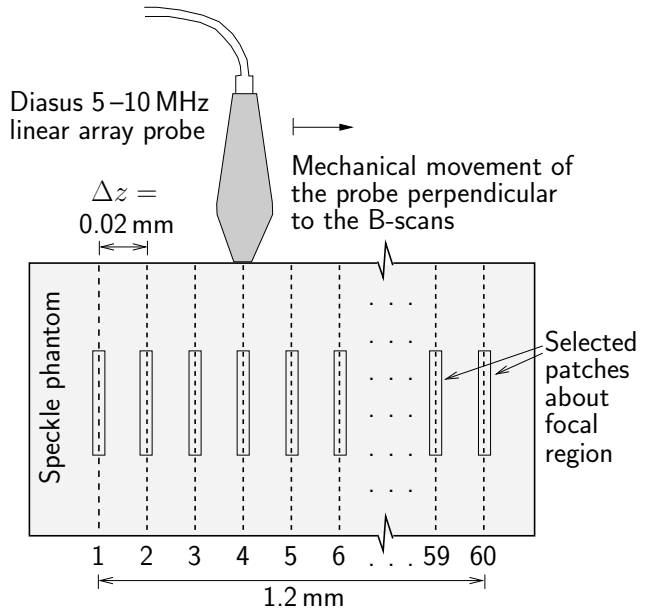

60 B-scans acquired with equidistant elevational spacing

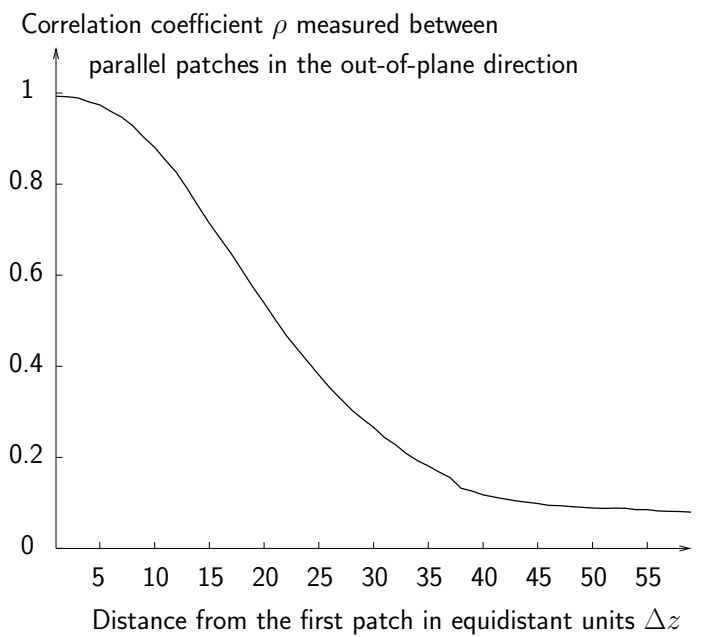

Figure 2: Example of a speckle decorrelation curve in the elevational direction. Left: Illustration of the experimental setup indicating the movement of the probe perpendicular to the B-scans (note the modified proportions for drawing purposes). Right: Measured decorrelation curve for a patch about the focus with respect to the first patch as fixed reference.

separation. Four speckle detectors are presented in Section 3: all are theoretically motivated, two of them are novel and two of them have been reported before [10, 25]. In Section 4, we evaluate the utility of the speckle detectors by estimating the out-of-plane separation of in vitro $\mathrm{RF}$ data acquired from a joint of beef. We present the main conclusions of the paper in Section 5.

\section{Local model of the resolution cell}

\subsection{Experimental apparatus}

For all experiments in this paper, a Diasus US machine ${ }^{2}$ modified for RF acquisition was used with a $5-10 \mathrm{MHz}$ linear array probe. The US machine was set up to acquire images at a $4 \mathrm{~cm}$ depth setting with a single focus at $2 \mathrm{~cm}$. After time gain compensation, the RF data were digitised using a 14 bit Gage CompuScope CS14100 ${ }^{3}$ analogue to digital converter. Whole frames, each comprising $127 \mathrm{RF}$ vectors by 3818 samples, were stored in on-board Gage memory before transferring to PC memory at $75 \mathrm{MB} / \mathrm{s}$. The system operates in real time, with acquisition rates exceeding 30 frames per second. Sampling was at $66.67 \mathrm{MHz}$, synchronous with the ultrasound machine's internal clock: this synchronization minimises phase jitter between vectors. Assuming an average speed of sound of $1540 \mathrm{~m} / \mathrm{s}$ in soft tissue, the acquired RF samples have an axial resolution of $\Delta y=0.01 \mathrm{~mm}$. The RF vectors were filtered with a $3-30 \mathrm{MHz}$ broadband filter, yielding an echo signal which we shall refer to as

\footnotetext{
${ }^{2}$ Dynamic Imaging Ltd., Cochcrane Square, Brucefield Industrial Park, Livingston EH54 9DR, Scotland, UK, http://www.dynamicimaging.co.uk/

${ }^{3}$ Gage Applied, Inc., 32nd Avenue, Lachine, QC, H8T 3H7, USA, http://www.gage-applied.com/
} 
$r(t)$. The instantaneous amplitude (the so called A-line) is obtained via envelope detection

$$
e(t)=|r(t)+j \hat{r}(t)|,
$$

where $\hat{r}(t)$ denotes the Hilbert transform of $r(t)$. The expression $r(t)+j \hat{r}(t)$ is referred to as the analytic signal or complex RF echo. The echo intensity is the square of $e(t)$.

Due to the coloured nature of the received RF echo, it is possible to estimate the local shape of the resolution cell via the spatial decorrelation of the RF echo intensity. For scans comprising fully developed speckle, there is a direct relationship between the resolution cell's dimensions and the decorrelation functions in the principal scanning directions $[2,5,31,32]$. We therefore measured decorrelation functions in the elevational and axial directions by scanning a speckle phantom with Rayleigh backscatter and uniform attenuation of $0.4 \mathrm{~dB} / \mathrm{cm} / \mathrm{MHz}^{4}[17,19]$. The probe was mounted on a mechanical assembly such that it could be translated slowly in the elevational direction by means of a screw thread. Its displacement was measured using a dial gauge. In an initial experiment, sixty parallel slices of RF data were recorded at distances $\Delta z=0.02 \mathrm{~mm}$ apart, as depicted in the left part of Fig. 2. To illustrate a typical decorrelation curve, consider a rectangular patch comprising approximately $5000 \mathrm{RF}$ samples about the focal region. The echo intensity was correlated with 59 corresponding patches with shifted ranges in the elevational and axial directions, as shown in the left halves of Figs. 2 and 3. The two decorrelation curves are depicted in the respective right halves of the figures. For the patch chosen in this example, the lengths of the principal axes of the roughly ellipsoidal resolution cell (measured at $-3 \mathrm{~dB}$ of the decorrelation curves) were approximately $0.98 \mathrm{~mm}$ in the elevational direction and $0.22 \mathrm{~mm}$ in the axial direction. Both elevational and axial decorrelation play a key role in this study. The elevational decorrelation is required for out-of-plane distance estimation, while the axial decorrelation is exploited by some speckle detectors.

\subsection{Training of local decorrelation curves}

100 frames of RF data were recorded from the speckle phantom, as described in Sec. 2.1, with the same probe settings but at a pitch of $0.04 \mathrm{~mm}$ in the elevational direction. Monte Carlo simulations in our previous work have indicated that meaningful speckle statistics can be estimated with patch sizes of about $5000 \mathrm{RF}$ samples. We therefore partitioned each frame of $\mathrm{RF}$ data into a grid of $8 \times 12$ rectangular disjoint patches, each comprising about 5000 RF samples. For each patch of the grid, we trained a model of its axial decorrelation using Pearson's linear correlation coefficient $\rho$ :

$$
\rho(X, Y)=\frac{N \sum x_{i} y_{i}-\sum x_{i} \sum y_{i}}{\sqrt{\left[N \sum x_{i}^{2}-\left(\sum x_{i}\right)^{2}\right]\left[N \sum y_{i}^{2}-\left(\sum y_{i}\right)^{2}\right]}},
$$

where $X=\left\{x_{i}\right\}$ and $Y=\left\{y_{i}\right\}, i=1 \ldots N$, are two patches of RF intensity values $x_{i}$ and $y_{i}$ respectively.

A single Gaussian (Eq. 4) with parameter $\alpha$ was chosen to model the decorrelation:

$$
\rho_{\mathrm{m}}=\exp \left(\frac{-\delta^{2}}{\alpha^{2}}\right)
$$

\footnotetext{
${ }^{4}$ Dept. of Medical Physics, University of Wisconsin-Madison, Madison, WI, USA
} 

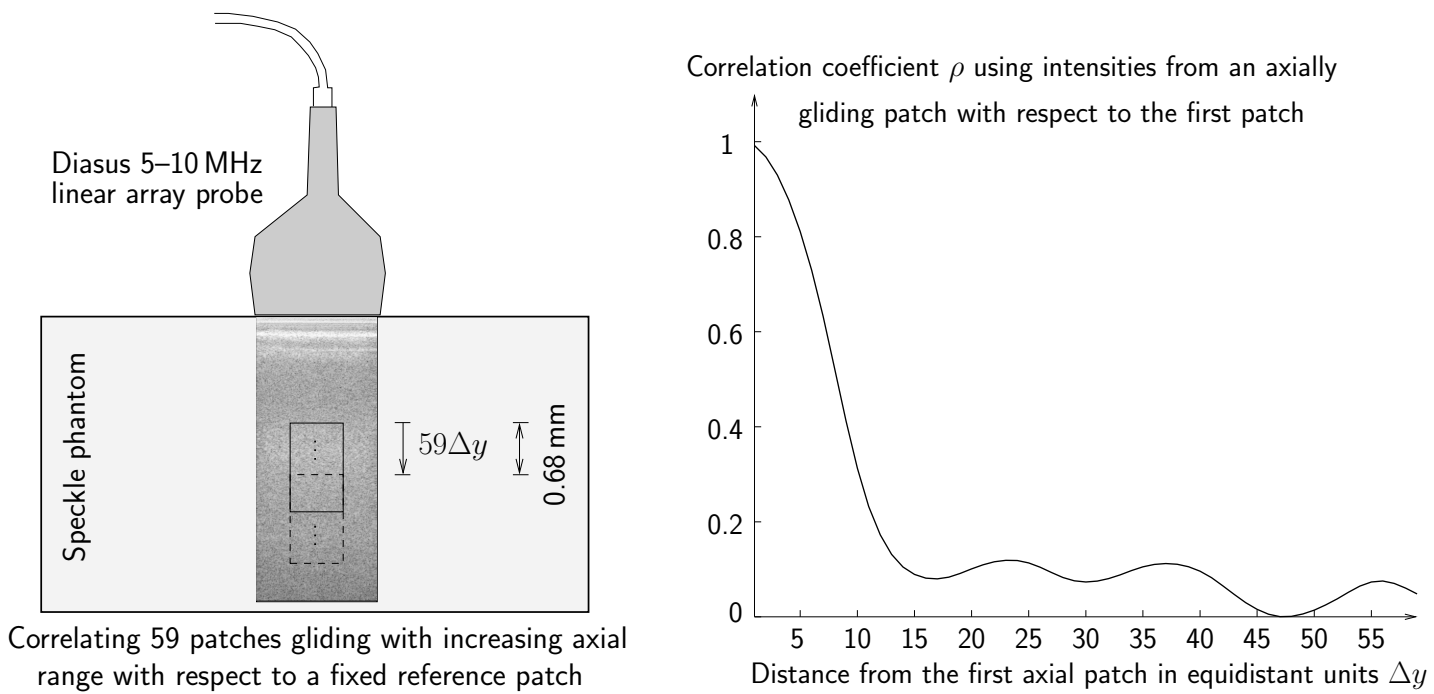

Figure 3: Example of a speckle decorrelation curve in the axial direction. Left: A rectangular patch about the focal region is correlated with corresponding patches within the same slice moved successively downwards in unit steps of one RF sample $(\Delta y=0.01 \mathrm{~mm})$. Right: Measured decorrelation curve with respect to the first patch.

where $\rho_{\mathrm{m}}$ is the correlation at distance $\delta$. We trained the axial model only for correlation values above 0.65 , where the measured curves could be approximated satisfactorily with a single Gaussian. For each patch, 100 decorrelation curves were obtained, one from each frame in the data set. These were averaged together, and the Gaussian model fitted to the averaged data. A linear least squares fit of the logarithmic (and thus linearised) model was obtained by minimising the sum of the squared deviations at $n$ samples of the decorrelation curve:

$$
\text { minimise : } \quad R^{2}=\sum_{i=1}^{n}\left[\ln \rho_{i}+\frac{\delta_{i}^{2}}{\alpha^{2}}\right]^{2}
$$

The necessary condition for a minimum is $\frac{\partial\left(R^{2}\right)}{\partial \alpha}=0$, from which it is readily shown that

$$
\alpha^{2}=-\frac{\sum_{i=1}^{n} \delta_{i}^{4}}{\sum_{i=1}^{n} \delta_{i}^{2} \ln \rho_{i}}
$$

The averaged axial decorrelation curves for each patch, together with an overlay of the fitted Gaussian models, are depicted in Fig. 4. Note that the Gaussian models do not fit all decorrelation curves accurately. At the top of the B-scan, this is due to the phantom's scanning window introducing strong specular features, as is evident in the example at the bottom left of Fig. 6. At the bottom of the B-scan, the problem is noise in the RF data due to increased attenuation. Hence, only those patches in rows 3 to 8 were used in the in vitro experiment reported in Sec. 4.

A similar experiment was conducted for the purpose of training models of the local speckle decorrelation in the elevational direction. For each patch in frame $f$, an elevational decorrelation curve was obtained by correlating with the corresponding patch in frame $f+i$, 

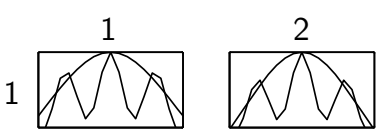

$2 \square$
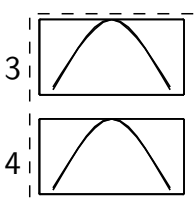

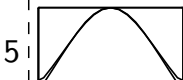
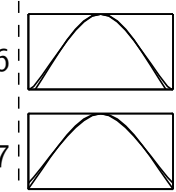

8:
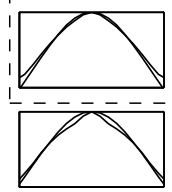

10

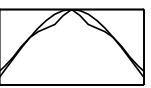

11

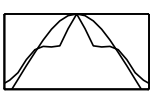

12

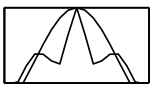

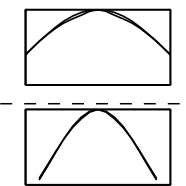
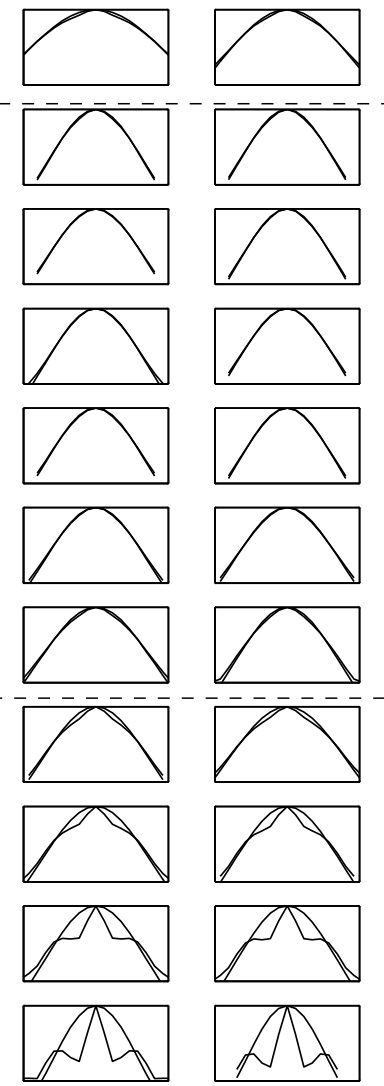
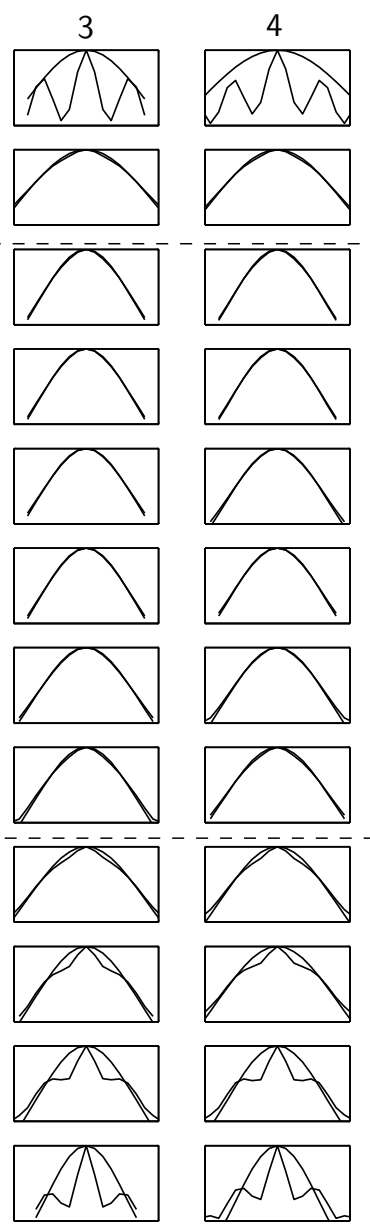
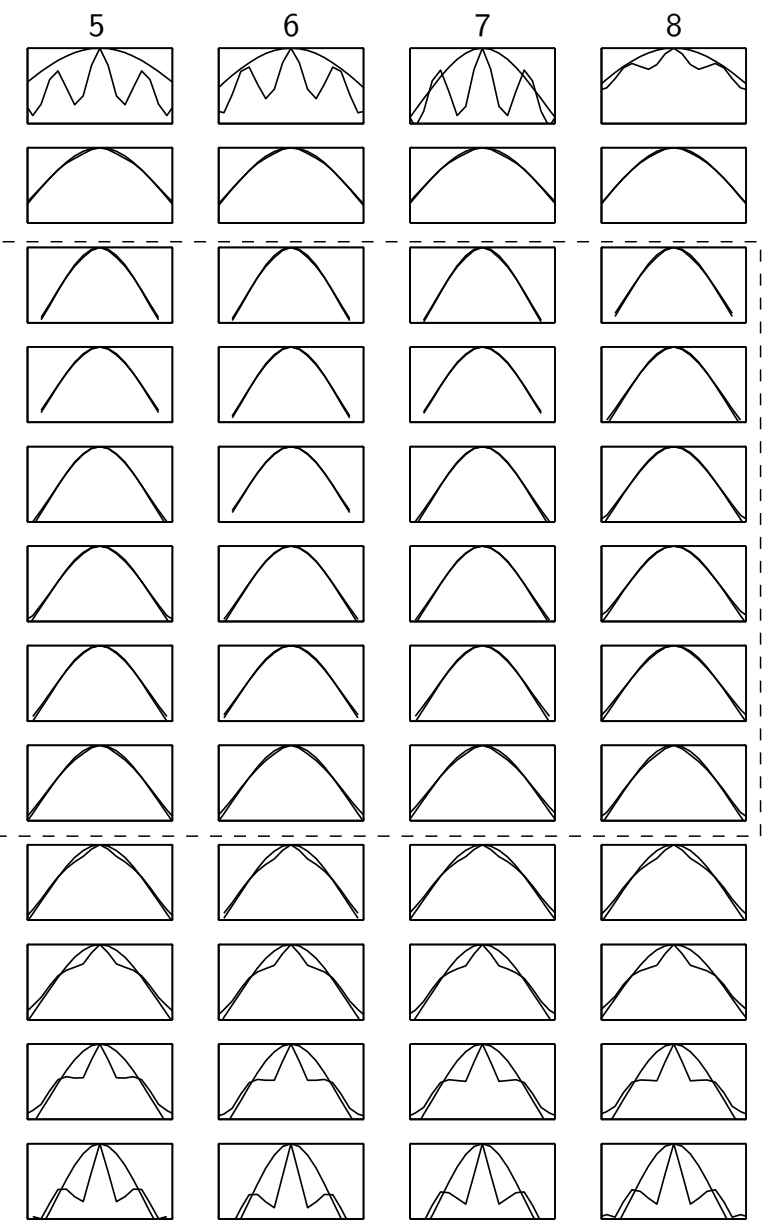

Figure 4: Decorrelation curves in the axial direction together with an overlay of the corresponding fitted Gaussian models for a grid of $8 \times 12$ rectangular patches. The models were trained using echo intensities from $100 \mathrm{RF}$ scans. The data set was recorded from a speckle phantom using the Diasus $5-10 \mathrm{MHz}$ probe at a $4 \mathrm{~cm}$ depth setting with a single focus at $2 \mathrm{~cm}$. The abscissae range from $-0.1 \mathrm{~mm}$ to $0.1 \mathrm{~mm}$. The correlation values on the ordinates range from 0.45 to 1 . Only the patches in rows 3 to 8 were selected for the later in vitro experiment. See the text for further experimental details. 
$i=1 \ldots 12$. An average decorrelation curve was calculated by averaging the 88 individual curves for $f=1 \ldots 88$. The Gaussian model was then fitted to the average curve, one for each patch. The averaged elevational decorrelation curves, together with an overlay of the Gaussian models, are depicted in Fig. 5. As with the axial model, only those patches within the dashed rectangle were chosen for the in vitro experiment reported in Sec. 4.

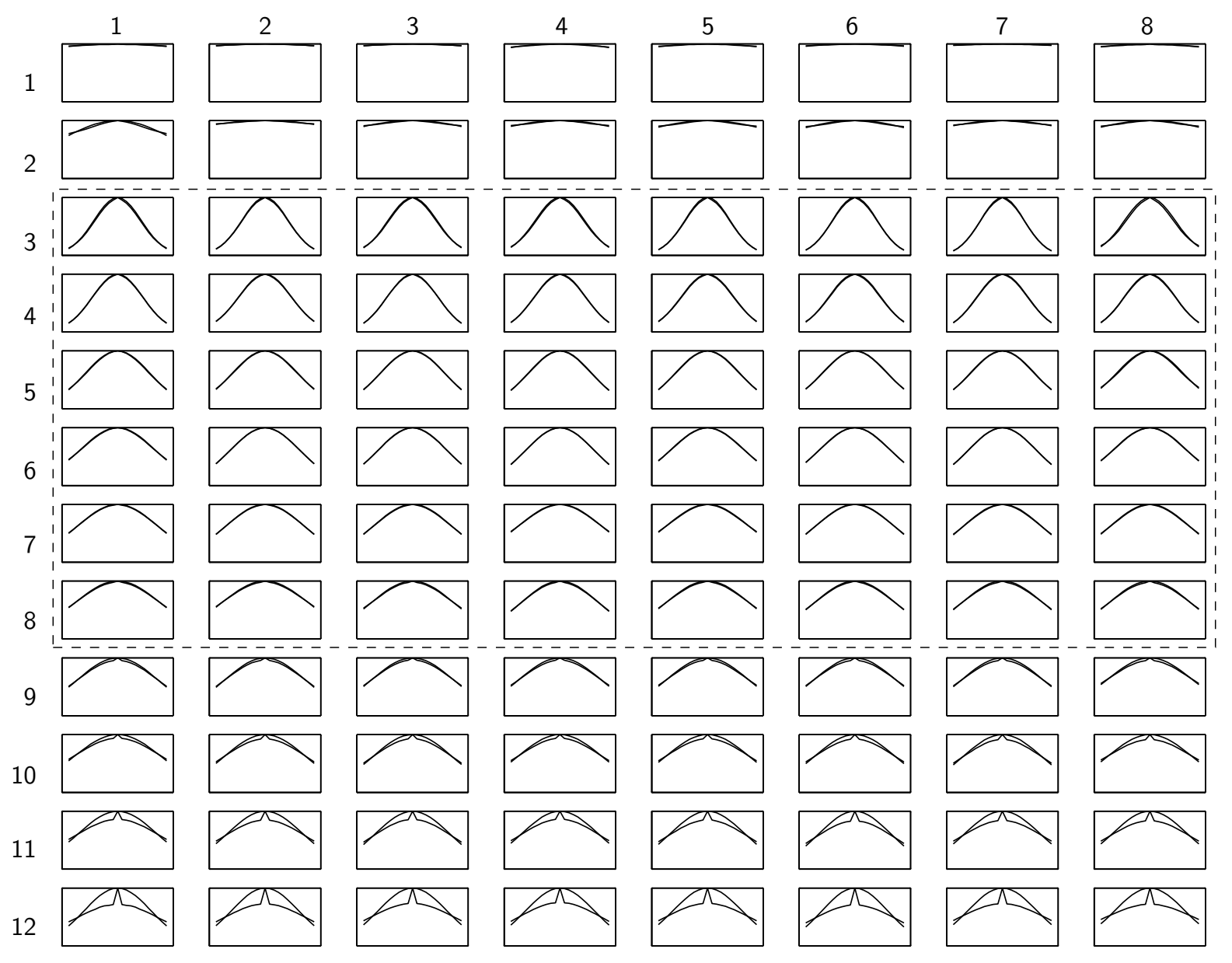

Figure 5: Decorrelation curves in the elevational direction together with an overlay of the corresponding fitted Gaussian models for a grid of $8 \times 12$ rectangular patches. The models were trained using echo intensities from 100 parallel $\mathrm{RF}$ scans $0.04 \mathrm{~mm}$ apart. The data set was recorded from a speckle phantom using the Diasus $5-10 \mathrm{MHz}$ probe at a $4 \mathrm{~cm}$ depth setting with a single focus at $2 \mathrm{~cm}$. The abscissae range from $-0.5 \mathrm{~mm}$ to $0.5 \mathrm{~mm}$. The correlation values on the ordinates range from 0 to 1 . Only the patches in rows 3 to 8 were selected for the later in vitro experiment. See the text for further experimental details. 


\section{Approaches to speckle detection}

Speckle detection is useful to aid segmentation, support diagnosis via quantitative tissue characterisation, for adaptive speckle suppression algorithms and, in the context of this report, as a prerequisite for speckle-based distance measurement. Fully developed speckle results from many diffuse scatterers per resolution cell. In this case, the underlying linear system theory describes the interaction of the propagating pulse with the scatterers as a summation of many random variables. The central limit theorem (CLT) states that data which are influenced by many small and unrelated random effects are approximately Normally distributed. Since this is the case for fully developed speckle, the received back-scattered RF echo values are Normally distributed, their corresponding envelope values (instantaneous amplitudes) are Rayleigh distributed, and their corresponding brightness values (instantaneous intensities) have an exponential distribution [30].

The following subsections theoretically motivate the four approaches to speckle detection as employed in the in vitro experiment in Sec. 4.

\subsection{Agreement of regression and correlation}

For fully developed speckle, Prager et al. derived the numerical equality of the regression parameter $b$ (the gradient of the regression line) and Pearson's correlation coefficient $\rho$ for uncompressed RF echo envelope intensity samples separated in the elevational direction [26]. To employ this result for speckle detection, we assume that if $b$ and $\rho$ differ, this difference is likely to result from coherent scattering or noise. We used the symmetric linear regression technique, as described in [26], to work out $b$ for two patches of echo envelope intensities. The corresponding linear correlation coefficient $\rho$ was calculated according to Eq. (3) using the same intensity values.

\subsection{Agreement of the observed axial decorrelation with its model}

As illustrated in Fig. 4, we trained local models of the axial decorrelation from scans of a speckle phantom. However, the shape of the decorrelation curves may be altered in scans of biological tissue due to coherent scattering. If the examined volume contains few scatterers, the diameter of the scatterers is wider than the interrogating pulse width (resolvable scatterers), or given a periodic arrangement of the scatterers, higher correlation values are observed than would be expected from fully developed speckle. Therefore, we assume that if there is a large enough deviation of the observed axial decorrelation curve from the trained model for that patch, then this difference is caused by coherent scattering or noise. The deviation is measured as the root mean square (RMS) difference between the observed decorrelation curve and the corresponding local model.

\subsection{Moment-based first order statistics classification}

Prager et al. $[22,23,25]$ extended the approach of Dutt and Greenleaf for speckle detection using first order statistics [6, 7]. Tuples of mean over standard deviation $(R)$ and the skewness statistics $(S)$ calculated from patches of uncompressed echo intensity were used to detect speckle in $(R, S)$-space [25]. In this report, we use a generalised version of the elliptic region of acceptance reported in [22]. To yield a one-dimensional measure $\vartheta$ for classification purposes, we worked out the distance $d$ of the $(R, S)$-point to the centre of the ellipse. $\vartheta$ was then 
calculated as the ratio of $d$ to the radius $r$ of the ellipse in the point's direction. By this means, the elliptical nature of the feature space is preserved in the one-dimensional measure $\vartheta$.

\subsection{Non-parametric goodness of fit test}

For fully developed speckle, the received complex RF echo at any point is a circularly symmetric Gaussian random variable (RV) with the real and imaginary parts independent, zeromean Gaussian RV's with the same variance [1, 3, 30, 31]. As mentioned earlier, under these conditions, the phasor magnitudes (i.e. the echo amplitudes as given by Eq. 2) are Rayleigh distributed and the squared phasor magnitudes (intensities) are exponentially distributed [30].

This important result can be used to apply a formal statistical test, whether a given sample of RF echo values may be considered as Normally distributed, and thus fully diffuse, or not. For this purpose, Georgiou and Cohen propose the Kolmogorov-Smirnov nonparametric goodness of fit test (K-S test) [10]. The K-S test allows one to examine the diffuse scattering assumption directly from the empirical cumulative distribution function (ecdf) $P_{n}(w)$ of the sampled RF echo $r(t)$, rather than testing parameters derived from the distribution (e.g. the signal-to-noise ratio or a set of moments) $[7,20]$. The K-S test uses the maximum vertical distance between the ecdf $P_{n}(w)$ and its corresponding Normal distribution $G(w)$ with parameters $\hat{\mu}$ and $\hat{\sigma}$ estimated from the ecdf. This is a sensitive measure for the discrepancy between the two distributions. The K-S test statistic $D_{n}$ is given by

$$
D_{n}=\sup _{-\infty<w<\infty}\left|P_{n}(w)-G(w)\right| .
$$

Given sample sizes $n>40$, the widely used 0.95 and 0.99 quantiles for two-sided hypothesis testing can be approximated for the K-S test statistics $D_{n}$ by $1.36 n^{-1 / 2}$ and $1.63 n^{-1 / 2}$ respectively [4]. That is, any $D_{n}$, as defined by Eq. (7), which is equal to or greater than the 0.95 quantile for that $n$ will be regarded as significant at the 0.05 level. Further details and an implementation of the K-S test can be found in [27].

\subsection{Verification of speckle detectors}

We verified the operation of the moment-based and K-S detectors (Sects. 3.3 and 3.4) using simulated 2D RF echo data. We were unable to test the other detectors in this manner, since the regression/correlation test requires 3D data, while the axial model test needs training for a specific transducer. The Field II package ${ }^{5}$ was used to simulate RF echo samples from a virtual cyst phantom [15] for a transducer with 128 array elements, using $10^{5}$ diffuse scatterers in a cuboid volume of $60 \times 40 \times 15 \mathrm{~mm}^{3}$. Besides diffuse scattering in the background, the resulting B-scan (Fig. 6, top) comprised three columns of (from left to right) five point targets, five strongly reflecting regions, and five cyst regions with varying diameter. The overlaid grid indicates the results of the speckle detection. Patches with $\vartheta<3$ were regarded as comprising speckle, and 0.05 was used as the significance level for the K-S test. Borders dashed in black and white indicate patches for which the two detectors agreed on speckle. White crosses indicate patches for which the two detectors agreed on non-speckle. Note how both detectors are reliably able to reject patches which clearly do not contain fully developed speckle. Next, we ran the two speckle detectors, with the same thresholds, on a B-scan from the speckle

\footnotetext{
${ }^{5}$ Field II Ultrasound Simulation Program, Technical University of Denmark, Biomedical Engineering Group, DK-2800 Kgs. Lyngby, Denmark, http://www.es.oersted.dtu.dk/staff/jaj/field/
} 

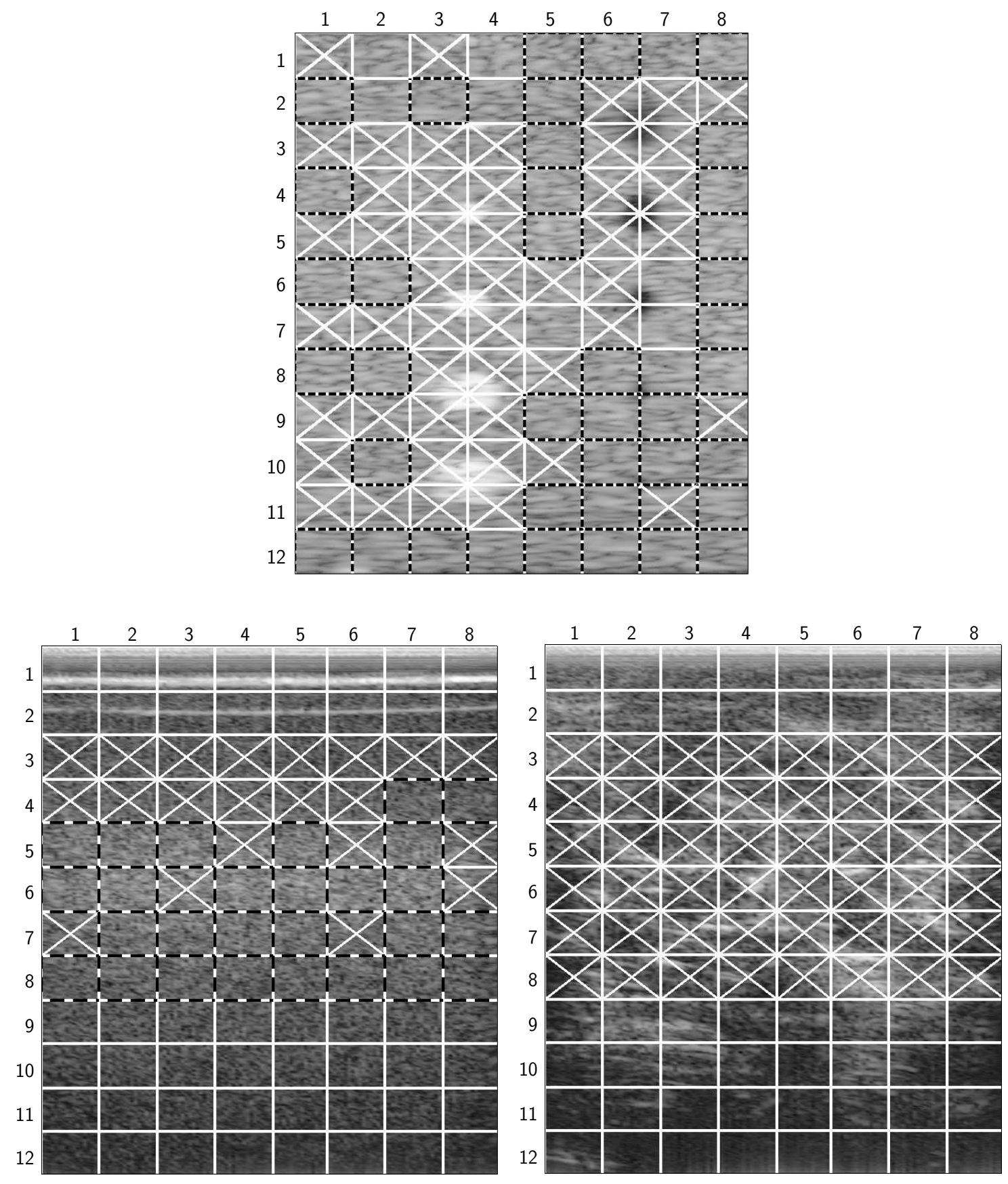

Figure 6: Typical B-scans and the results of speckle detection. The dashed frames indicate patches for which the two speckle detectors in Sects. 3.3 and 3.4 agreed on fully developed speckle, while the crosses indicate patches for which they agreed on non-speckle. Top: Speckle detection using the virtual cyst phantom simulated with Field II. Bottom left: First slice of the in vitro RF data set of the speckle phantom used for the training of the decorrelation curves. Bottom right: First slice of the in vitro RF data set of a beef joint used for the main experiment in Sec. 4. As indicated in Figs. 4 and 5, only patches in rows 3 to 8 were examined in the two in vitro data sets. Refer to Sec. 4.1 for experimental details. 
phantom (Fig. 6, bottom left). Note how both detectors agree on fully developed speckle for the majority of the patches.

\section{Accuracy of distance measurement using in vitro RF data}

In this section, we seek to address both the achievable accuracy of speckle-based out-of-plane distance measurement in soft tissue, and ways to improve it using speckle detection. To this end, we chose to perform an in vitro experiment. In vivo experiments are subject to additional factors that degrade speckle-based distance measurement, including the movement of the soft tissue due to breathing, muscular tremor, circulatory motion and the contact pressure of the probe itself [28].

\subsection{Experimental setup and methodology}

$\mathrm{RF}$ data was recorded from a joint of beef using the same settings as described in Sec. 2. A total of 100 parallel slices of RF data, $\Delta z=0.04 \mathrm{~mm}$ apart, was recorded. The first slice of the beef data is shown at the bottom right of Fig. 6. The speckle detector thresholds were the same as adopted for the other two B-scans. Note how none of the data is classified as fully developed speckle at these thresholds.

We measured distances in the beef and training data sets at an elevation separation of $0.20 \mathrm{~mm}$ (every fifth slice). For the reasons outlined in Sec. 2, we limited the measurements in each frame to the patches in rows 3 to 8 . Frames $z \in\{1, \ldots, 95\}$ were chosen successively as reference frames. Speckle-based distance measurement was carried out by comparing selected patches with their corresponding patches in frame $z+5$, using the local model of the elevational decorrelation trained in Sec. 2.2. For each pair of corresponding patches $0.20 \mathrm{~mm}$ apart, the following values were recorded:

- the linear regression gradient $b$ between the $\mathrm{RF}$ echo envelope intensity values of the corresponding patches,

- Pearson's linear correlation coefficient $\rho$ between the RF echo envelope intensity values of the corresponding patches,

- the measured elevational distance between the patches using Eq. 4,

- the root mean square (RMS) errors characterising how well each patch fitted its axial model,

- the tuples of $(R, S)$ moments for each patch (using uncompressed RF echo envelope intensities),

- the K-S test statistics $D_{n}$, calculated from the real RF echo samples $r(t)$, measuring the deviation of the distribution of the RF samples from a fitted Normal distribution (for each patch).

For each speckle detector, the threshold $\tau$ was varied to observe its effect on elevational distance measurement. 
- Detector $\mathrm{D}_{1}$ measured the agreement of regression and correlation (see Sec. 3.1). The speckle hypothesis was accepted if:

$$
|b-\rho| \leq \tau_{1}
$$

- Detector $\mathrm{D}_{2}$ measured the agreement of the observed axial decorrelation with its trained model (see Sec. 3.2) for each pair of patches 1 and 2 separated by $\Delta z=0.20 \mathrm{~mm}$. The speckle hypothesis was accepted if:

$$
\max \left(\mathrm{RMS}_{1}, \mathrm{RMS}_{2}\right) \leq \tau_{2} .
$$

- Detector $\mathrm{D}_{3}$ used the feature $\vartheta$ (see Sec. 3.3) calculated for both patches 1 and 2. The speckle hypothesis was accepted if:

$$
\max \left(\vartheta_{1}, \vartheta_{2}\right) \leq \tau_{3} .
$$

- Detector $\mathrm{D}_{4}$ employed the the K-S test statistics $D_{n}$ (see Sec. 3.4) for patches 1 and 2. The speckle hypothesis was accepted if:

$$
\max \left(D_{n 1}, D_{n 2}\right) \leq \tau_{4}
$$

For evaluation purposes, the thresholds $\tau_{1}, \ldots, \tau_{4}$ were extended incrementally from zero until every patch was accepted as speckle. For each threshold value, the mean relative distance error and its variance were calculated from those pairs of patches for which the speckle hypothesis was accepted according to Eqs. (8)-(11).

\subsection{Results}

Figure 7 shows the frequency of the per-patch distance estimates without speckle detection. Unsurprisingly, the results for the training data are nearly perfect, with an average measured distance of $\bar{\delta}=0.204 \mathrm{~mm}$ and a standard deviation of $\sigma_{\delta}=0.022 \mathrm{~mm}$. The residual $2 \%$ overestimation can be explained by the locally imperfect fit of the Gaussian models with the averaged decorrelation curves. In marked contrast, note the significant bias of the beef results towards smaller distances. For all pairs of patches $0.20 \mathrm{~mm}$ apart, the average measured distance was $\bar{\delta}=0.134 \mathrm{~mm}$ (mean relative distance error $\bar{\epsilon}=-33.1 \%$ ) with a standard deviation of $\sigma_{\delta}=0.046 \mathrm{~mm}$. The coherent scattering in the beef is decorrelating slower than the speckle in the phantom, and this is misinterpreted systematically as a smaller separation between the patches. Occasional overestimates result from noisy patches towards the bottom of the B-scans.

The impact of the four speckle detectors on the quality of the distance measurements is shown in Fig. 8. The detector $\mathrm{D}_{1}$ was unable to improve the quality of the distance measurement. An examination of those pairs of patches with large distance error despite similar $b$ and $\rho$ values revealed that they had both large values of $\vartheta$ and a non-Gaussian statistic of the RF samples. We conclude that fully developed speckle is a sufficient, but not a necessary, condition for the equality of $b$ and $\rho$. For this reason, it is not possible to use $\mathrm{D}_{1}$ for speckle detection.

At first sight, the detector $\mathrm{D}_{3}$ appears to be most useful, improving the quality of the mean measured distance to $0.180 \mathrm{~mm}(-10.3 \%)$ and a standard deviation of $\pm 0.040 \mathrm{~mm}$ for values of 

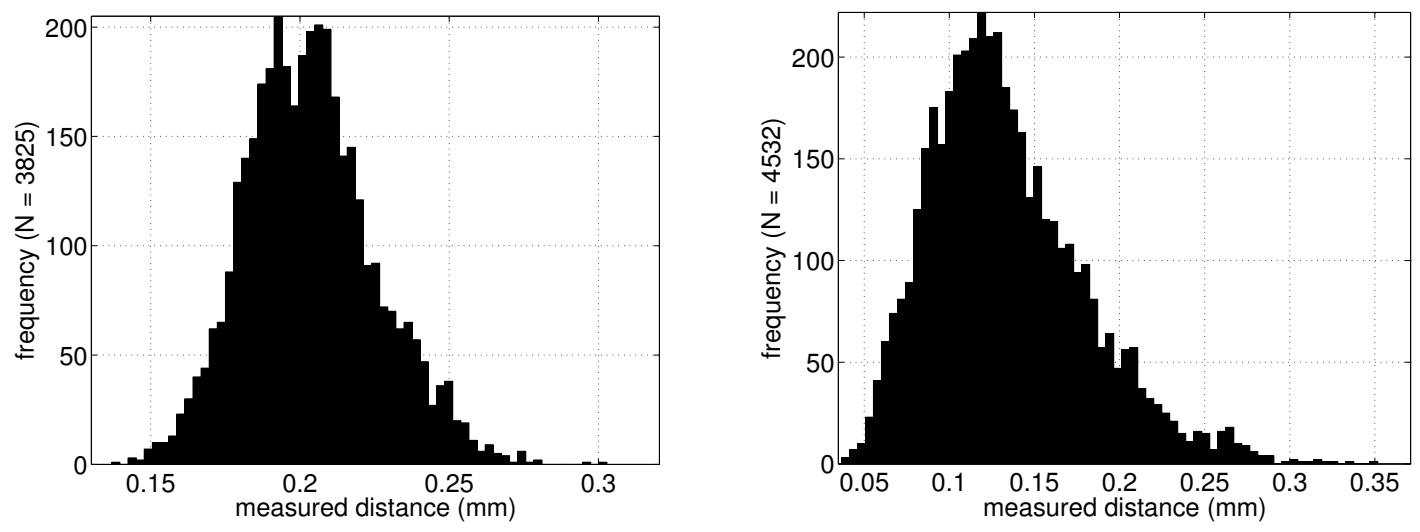

Figure 7: Histograms of the measured elevational distances for left the training data (mean $\hat{\mu}=0.204 \mathrm{~mm}$, standard deviation $\hat{\sigma}=0.022 \mathrm{~mm}$, skewness $\hat{\gamma}_{1}=0.376$ ) and right the beef data $(\hat{\mu}=0.134 \mathrm{~mm}, \hat{\sigma}=0.046 \mathrm{~mm}, \hat{\gamma}=0.799)$ for all pairs of patches with $\rho>0.65$ at a spacing of $\Delta z=0.20 \mathrm{~mm}$ apart.

$\vartheta<3.5$. However, this result is irrelevant with respect to sensorless freehand 3D ultrasound, since, at this threshold, there remain only 17 pairs of patches in the whole data set. At least three speckled patches per B-scan are required for a 3D reconstruction of the insonified volume. Therefore, we compared the achievable accuracy at the minimum thresholds such that, on average, at least three patches per B-scan were accepted as coming close to speckle according to $\mathrm{D}_{2}-\mathrm{D}_{4}$ (see Tab. 1). At these thresholds, the average relative underestimation of the elevational distances of $-33.1 \%$ could be improved to about $-25 \%$ using any of the detectors $\mathrm{D}_{2}-\mathrm{D}_{4}$. The detectors $\mathrm{D}_{2}$ and $\mathrm{D}_{3}$ performed slightly better than $\mathrm{D}_{4}$.

Table 1: Descriptive statistics of the distance measurements at thresholds $\tau$ where, on average, and at least, three patches per B-scan were accepted according to the speckle detectors $\mathrm{D}_{2}-\mathrm{D}_{4}$.

\begin{tabular}{llll}
\hline patches per B-scan & \multicolumn{1}{c}{$\mathrm{D}_{2}$} & \multicolumn{1}{c}{$\mathrm{D}_{3}$} & \multicolumn{1}{c}{$\mathrm{D}_{4}$} \\
\hline three on average at & $\tau_{2}=0.1076(n=285)$ & $\tau_{3}=5.6508(n=285)$ & $\tau_{4}=0.0380 \quad(n=285)$ \\
$\left(\bar{\delta} \pm \sigma_{\delta}\right)[\mathrm{mm}]$ & $0.150 \pm 0.045$ & $0.149 \pm 0.037$ & $0.148 \pm 0.034$ \\
$\left(\bar{\epsilon} \pm \sigma_{\epsilon}\right)[\%]$ & $-25.3 \pm 22.7$ & $-25.7 \pm 18.4$ & $-26.0 \pm 16.8$ \\
\hline at least three at & $\tau_{2}=0.7484(n=667)$ & $\tau_{3}=7.2769(n=664)$ & $\tau_{4}=0.0467 \quad(n=540)$ \\
$\left(\bar{\delta} \pm \sigma_{\delta}\right)[\mathrm{mm}]$ & $0.141 \pm 0.047$ & $0.144 \pm 0.040$ & $0.1382 \pm 0.035$ \\
$\left(\bar{\epsilon} \pm \sigma_{\epsilon}\right)[\%]$ & $-29.3 \pm 23.7$ & $-27.9 \pm 19.9$ & $-30.9 \pm 17.5$ \\
\hline
\end{tabular}

\section{Conclusions}

In patches of $2 \mathrm{D}$ ultrasound data of biological tissue, there is little to no scattering that fits the model of "fully developed speckle". Almost all observed scattering is to some extent 

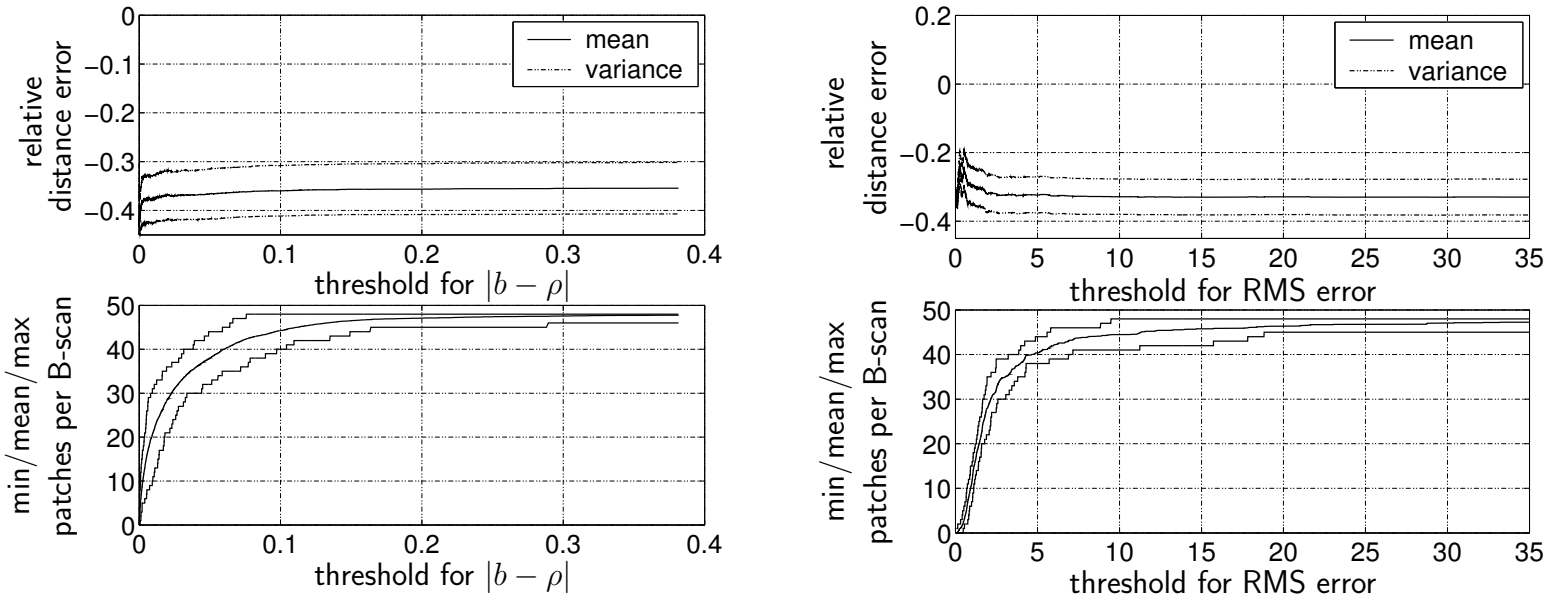

(a) results for D1

(b) results for D2
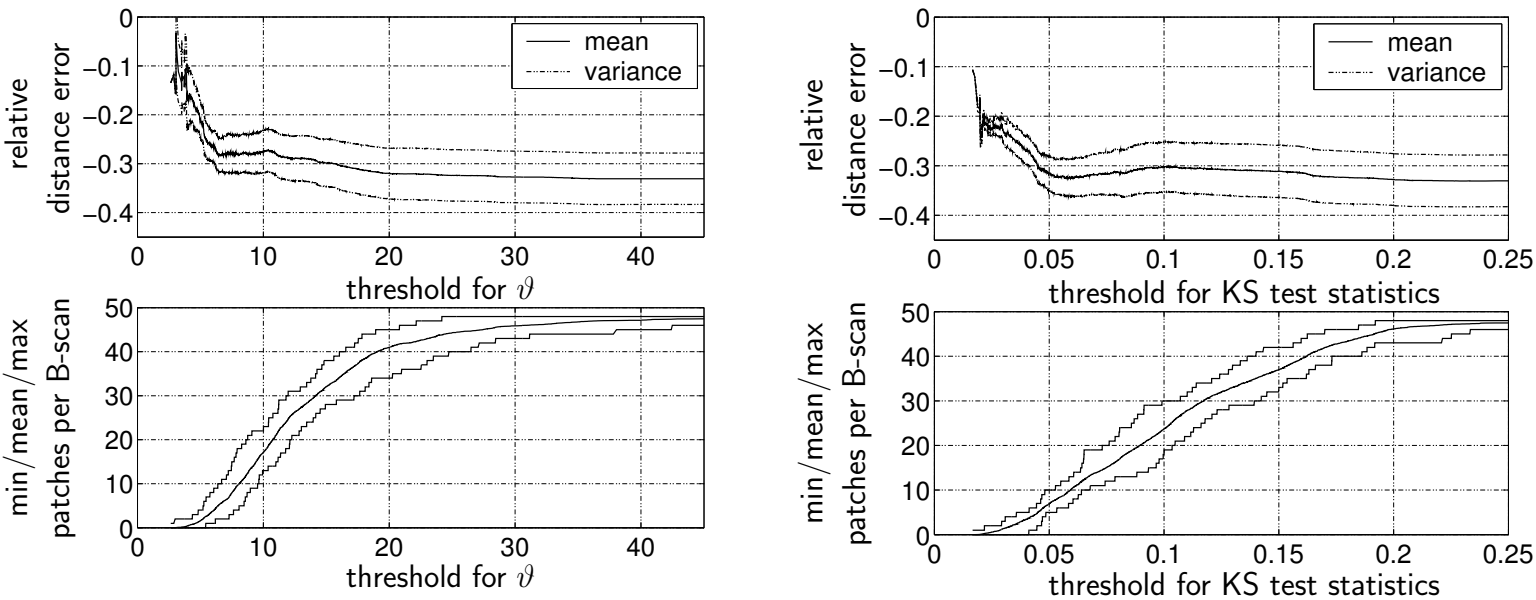

(c) results for D3

(d) results for D4

Figure 8: Incremental thresholding vs. the relative distance error and the number of patches remaining per B-scan. 
specular, i. e. has varying degrees of coherency. At least three accurate elevational distance measurements, at three linearly independent points per B-scan, are required for a full metric $3 \mathrm{D}$ reconstruction of a sweep of freehand ultrasound data. For this reason, speckle detection on its own is an insufficient means to obtain metric 3D reconstructions. However, speckle detection is still useful for improving qualitative $3 \mathrm{D}$ reconstructions by rejecting patches with strong specular scattering or noise.

\section{Acknowledgements}

This work was carried out under EPSRC grant number GR/S34366/01. Dynamic Imaging provided a modified Diasus ultrasound machine with direct access to the analogue RF signals. The authors would like to thank R. James Housden for his assistance in acquiring the RF data sets from the beef joint and the speckle phantom.

\section{References}

[1] C. B. Burckhardt. Speckle in ultrasound B-mode scans. IEEE Transactions on Sonics and Ultrasonics, SU-25(1):1-6, January 1978.

[2] J-F. Chen, J. B. Fowlkes, P. L. Carson, and J. M. Rubin. Determination of scan-plane motion using speckle decorrelation: theoretical considerations and initial test. International Journal of Imaging Systems Technology, 8:38-44, 1997.

[3] F. S. Cohen. Modeling of ultrasound speckle with application in flaw detection in metals. IEEE Transactions on Ultrasonics, Ferroelectrics and Frequency Control, 40(3):624-632, March 1992.

[4] W. J. Conover. Practical Nonparametric Statistics. John Wiley \& Sons, New York, 2nd edition, 1980.

[5] R. J. Dickinson and C. R. Hill. Measurement of soft tissue motion using correlation between A-scans. Ultrasound in Medicine and Biology, 8:263-271, 1982.

[6] V. Dutt and J. F. Greenleaf. Ultrasound echo envelope analysis using a homodyned k distribution signal model. Ultrasonic Imaging, 16:265-287, 1994.

[7] V. Dutt and J. F. Greenleaf. Speckle analysis using signal to noise ratios based on fractional order moments. Ultrasonic Imaging, 17:251-268, 1995.

[8] A. Fenster, D. B. Downey, and H. N. Cardinal. Three-dimensional ultrasound imaging. Physics in Medicine and Biology, 46:R67-R99, 2001.

[9] A. H. Gee, R. W. Prager, G. M. Treece, and L. H. Berman. Engineering a freehand 3D ultrasound system. Pattern Recognition Letters, 24(4-5):757-777, 2003.

[10] G. Georgiou and F. S. Cohen. Statistical characterization of diffuse scattering in ultrasound images. IEEE Transactions on Ultrasonics, Ferroelectrics and Frequency Control, 45(1):57-64, Jan. 1998. 
[11] P. Hassenpflug, R. W. Prager, G. M. Treece, and A. H. Gee. Distance measurement for sensorless 3D US. In Proceedings of 7th International Conference on Medical Image Computing and Computer-Assisted Intervention (MICCAI 2004), pages 1087-1088, Saint-Malo, France, September 2004. LNCS 3217, Part II, Springer.

[12] H. Hotelling. Relation between two sets of variates. Biometrika, 28:321-377, 1936.

[13] A. Hyvärinen, J. Karhunen, and E. Oja. Independent Component Analysis. Adaptive and Learning Systems for Signal Processing, Communications, and Control. John Wiley \& Sons, New York, 2001.

[14] J. A. Jensen. Field: A program for simulating ultrasound systems. In 10th NordicBaltic Conference on Biomedical Imaging, volume 34, Supplement 1, Part1 of Medical \& Biological Engineering \& Computing, pages 351-353, 1996.

[15] J. A. Jensen and P. Munk. Computer phantoms for simulating ultrasound B-mode and cfm images. In Proc. of the 23rd Acoustical Imaging Symposium, Boston, Massachusetts, USA, April 13-16 1997.

[16] I. T. Jolliffe. Principal Component Analysis. Springer Series in Statistics. Springer, New York, 2nd edition, 2002.

[17] J. M. Kofler Jr. and E. L. Madsen. Improved method for determining resolution zones in ultrasound phantoms with spherical simulated lesions. Ultrasound in Medicine and Biology, 27(12):1667-1676, 2001.

[18] M. Li. System and method for 3D medical imaging using 2D scan data., September 1995. United States patent 5,582,173. Application number 529778.

[19] E. L. Madsen, G. R. Frank, and F. Dong. Liquid or solid ultrasonically tissue-mimicking materials with very low scatter. Ultrasound in Medicine and Biology, 24(4):535-542, 1998.

[20] V. M. Narayanan, P. M. Shankar, and J. M. Reid. Non-Rayleigh statistics of ultrasonic backscattered signals. IEEE Transactions on Ultrasonics, Ferroelectrics and Frequency Control, 41:845-852, Nov. 1994.

[21] T. R. Nelson and D. H. Pretorius. Three-dimensional ultrasound imaging. Ultrasound in Medicine and Biology, 24(9):1243-1270, 1998.

[22] R. W. Prager, A. H. Gee, G. M. Treece, and L. H. Berman. Speckle detection in ultrasound images using first order statistics. Technical Report CUED/F-INFENG/TR 415, Cambridge University Engineering Department, July 2001.

[23] R. W. Prager, A. H. Gee, G. M. Treece, and L. H. Berman. Analysis of speckle in ultrasound images using fractional-order statistics of the homodyned k-distribution. Ultrasonics, 40(1-8):133-137, May 2002.

[24] R. W. Prager, A. H. Gee, G. M. Treece, and L. H. Berman. Freehand 3D ultrasound without voxels: volume measurement and visualisation using the stradx system. Ultrasonics, 40(1-8):109-115, May 2002. 
[25] R. W. Prager, A. H. Gee, G. M. Treece, and L. H. Berman. Decompression and speckle detection for ultrasound images using a homodyned $\mathrm{k}$ distribution. Pattern Recognition Letters, 24(4-5):705-713, 2003.

[26] R. W. Prager, A. H. Gee, G. M. Treece, C. J. C. Cash, and L. H. Berman. Sensorless freehand 3D ultrasound using regression of the echo intensity. Ultrasound in Medicine and Biology, 29(3):437-446, March 2003.

[27] W. H. Press, S. A. Teukolsky, W. T. Vetterling, and B. P. Flannery. Numerical Recipes in C. Cambridge University Press, Cambridge, UK, 1993.

[28] G. M. Treece, R. W. Prager, A. H. Gee, and L. H. Berman. Correction of probe pressure artifacts in freehand 3D ultrasound. Medical Image Analysis, 6(3):199-215, September 2002.

[29] T. A. Tuthill, J. F. Krücker, J. B. Fowlkes, and P. L. Carson. Automated threedimensional US frame positioning computed from elevational speckle decorrelation. $R a-$ diology, 209:575-582, 1998.

[30] R. F. Wagner, S. W. Smith, J. M. Sandrik, and H. Lopez. Statistics of speckle in ultrasound B-scans. IEEE Transactions on Sonics and Ultrasonics, 30(3):156-163, May 1983.

[31] R.F. Wagner, M. F. Insana, and D. G. Brown. Statistical properties of radio-frequency and envelope-detected signals with applications to medical ultrasound. Journal of the Optical Society of America A, 4(5):910-922, May 1987.

[32] R.F. Wagner, M. F. Insana, and S. W. Smith. Fundamental correlation lengths of coherent speckle in medical ultrasound images. IEEE Transactions on Ultrasonics, Ferroelectrics and Frequency Control, 35(1):34-44, January 1988.

[33] P. N. T. Wells and M. Halliwell. Speckle in ultrasonic imaging. Ultrasonics, 19(5):225229, September 1981. 